\title{
CORRECTION
}

\section{Correction: Inflammatory markers and bone mass in children with overweight/obesity: the role of muscular fitness}

Jose J. Gil-Cosano1, Luis Gracia-Marco ${ }^{1,2}$, Esther Ubago-Guisado ${ }^{1,3}$, Idoia Labayen ${ }^{4}$, Mireia Adelantado-Renau ${ }^{5}$, Cristina Cadenas-Sanchez ${ }^{1,6}$, Jose Mora-Gonzalez ${ }^{1}$, Abel Plaza-Florido ${ }^{1}$, Concepción M. Aguilera ${ }^{7,8}$, José Gómez-Vida ${ }^{9}$, José Maldonado $^{10,11}$, Jaak Jürimäe ${ }^{12}$ and Francisco B. Ortega ${ }^{1}$

Pediatric Research (2020) 87:179; https://doi.org/10.1038/s41390-019-0637-8

Correction to: Pediatric Research https://doi.org/10.1038/s41390-019-0572-8, published online 07 September 2019

In the original version of this article, the following funding source was accidentally omitted:
“European Regional Development Funds (ref. SOMM17/6107/UGR)”

This additional funding source has now been included in the Acknowledgements section. This has been corrected in both the PDF and HTML versions of the article.

\footnotetext{
"PROFITH "PROmoting FITness and Health Through Physical Activity" Research Group, Sport and Health University Research Institute (iMUDS), Department of Physical and Sports Education, Faculty of Sport Sciences, University of Granada, Granada, Spain; ${ }^{2}$ Growth, Exercise, Nutrition and Development Research Group, University of Zaragoza, Zaragoza, Spain; ${ }^{3}$ Health and Social Research Center, Universidad de Castilla-La Mancha, Cuenca, Spain; ${ }^{4}$ Institute for Innovation \& Sustainable Development in Food Chain (IS-FOOD), Public

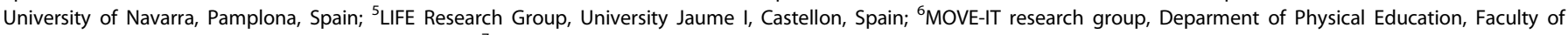
Education Sciences, University of Cádiz, Cádiz, Spain; ${ }^{7}$ Department of Biochemistry and Molecular Biology II, Institute of Nutrition and Food Technology, Centre for Biomedical Research, University of Granada, Granada, Spain; ${ }^{8} \mathrm{CIBER}$ Fisiopatología de la Obesidad y la Nutrición (CIBEROBN), Madrid, Spain; ${ }^{9}$ Department of Pediatrics, San Cecilio Hospital,

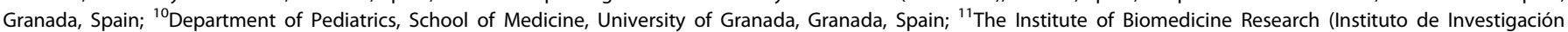
Biosanitaria (IBS), Granada, Spain and ${ }^{12}$ Institute of Sport Sciences and Physiotherapy, University of Tartu, 51007 Tartu, Estonia Correspondence: Jose J. Gil-Cosano (josejuangil@ugr.es)
}

Published online: 29 October 2019 